\title{
Women Safety Thread
}

\author{
R. Sharmila \\ Dept of ECE \\ SRM VEC \\ Kancheepuram, India \\ A.N. Ravindhar \\ Dept of ECE \\ SRM VEC \\ Kancheepuram, India
}

\author{
M. Saravanan \\ Dept of ECE \\ SRM VEC \\ Kancheepuram, India.
N. Usha Bhanu
Dept of ECE \\ SRM VEC \\ Kancheepuram, India
}

\begin{abstract}
In this present world women are less secure and have various issues regarding their security. To ensure their security we have proposed a module with copper thread, ATMEGA 328P, Global System for Mobile communication module, Global Positioning System (GSM) module, RF Transmitter and $\mathrm{RF}$ receiver, $\mathrm{HC}-05$ Bluetooth module and Comparator as their major component. In this proposed work we have two separate modules for transmitter and receiver. The transmitter module consists of copper thread which can be stitched along with the dress of the women which are not externally visible to the culprit. There will be a connection flow between these threads which is continuously monitored by the comparator. When the connection flow is terminated by breaking the copper thread, the GSM module makes emergency call and sends alert message along with the current location of the victim to the predefined numbers and the RF transmitter sends the signal to the RF receiver at the transmitter module. At the receiver module, when the $R F$ receiver receives the signal from the RF transmitter, it turns on the buzzer. At emergency cases the person themselves can break the thread to send alert messages.
\end{abstract}

Keywords-Women safety, Copper thread, Alert message, Current location, emergency call, not externally visible.

\section{INTRODUCTION}

Women in India the better half of Indian society, today, are becoming the most vulnerable section as far as their safety is concerned. When we read the pages of a newspaper, we come across many headlines reporting cases of sexual assault, molestation, sexual harassment, rapes, etc, this implies that there is an increasing trend of such sexual assaults in this present generation. To reduce the crime against women there are many devices and applications that have been proposed earlier. But usage of flex sensor and various other sensors as their major component reduced the usage of those applications and devices as there is a large chance of false alerts when the sensor value cross the fixed threshold value. So, to overcome this, we have proposed a method which has copper thread, GSM module, HS-O5 BLUETOOTH module, RF transmitter and receiver and ATMEGA 328P microcontroller as their major component. This method consists of two separate modules for transmitter and receiver. The copper thread can be stitched along with the dress of women a. There will be a connectivity flow between the threads. The transmitter consists of GSM and RF transmitter. GSM module sends alert message along with the location of the distress and makes emergency calls to the predefined numbers when the thread is broken by the victim and the RF transmitter sends signal to the receiver over RF network. The receiver module consists of RF receiver and buzzer. The RF receiver turns on the buzzer when it receives signal from the RF transmitter from RF network. At emergency cases the person themselves can break the thread to send alert message.

\section{LITERATURE SURVEY}

The literature survey describes about the papers that are studied and analyzed for the project. The following are the corresponding papers which defines existing women safety project. [1] The main purpose of this paper is to introduce a women's safety device. This device consists of a panic button which can be pressed by the victim when she is in danger. When the panic button is pressed alert message is sent to the predefined numbers along with the live location using GPS and GSM modules. It uses ATMEGA 328P microcontroller and embedded $\mathrm{C}$ language is used to interface the hardware components to the microcontroller. The alert message is sent in a loop with an interval of about 4 seconds. The device consists of three operation which is controlled using a mobile application. The first function is the default mode in this, when the device receives a message a call was initiated to the sender of the message. The second function is location send mode. This function is activated through a push button. In this, it sends the location of the victim as a hyperlink to the trusted contact through message. The third function is call making mode. This function is activated through a push button and it makes call to the trusted contact. [2] This paper proposes to have a device which is the integration of multiple device. This device consists of ATMEGA 2560 microcontroller, IoT modules, vibration sensor, neuro sensor and buzzer. This device is proposed as a wearable smart band which can be activated by the user when she holds on to the trigger button of the device. When the device is activated it sends the current location and alert message to the predefined numbers using GPS AND GSM modules. The neuro sensor is used to produce electric shock to the intruder. The major advantage of this system is its reduced size. [3] This paper proposes a safe and secured electronic system for women. The system consists of Arduino controller and various other sensors such as pulse rate sensor, temperature LM 35 sensor, flex sensor and sound sensor. The temperature sensor in this system is used to measure the body temperature of the woman. The pulse rate sensor is used to continuously monitor the pulse rate of the women. Flex sensor is used to measure the force due 
to harsh attack or touch. Sound sensor is used to measure the sound around the woman. When the threshold values of these sensor are crossed the system sends location of the victim to the predefined numbers using GPS and GSM. [4] This paper proposes a safety device which can be used to locate the missing child and also helps in the tracking of the child's movements outside the home. GSM and GPS are used to share the live location. The device is implemented using Raspberry pi model B. python programming is used to interface the hardware components to Raspberry pi module. The device is an easy to carry wearable device. The device has a voice recognition feature by which the user can activate the device through a specific keyword. It can be used by both children and women.

\section{PROPOSED SYSTEM ANALYSIS}

\section{A. Proposed Method}

In this present century a woman can be sexually attacked or raped at anytime, anywhere. Even at the work place they face various problems regarding their security. To provide security to the women outside their house is one of the greatest challenges of our current society. To provide a solution to this problem we have proposed a method which ensures the safety of women outside their house. The device gets started once the power supply is given. The device can be activated through Bluetooth, when a woman feels that she is under threat. Copper threads can be stitched along with the dress and the connection flow between the thread is continuously monitored by the comparator. Once the connection flow between the ends of the thread is terminated by breaking the thread, from the transmitter module emergency call and alert messages along with the location of the victim is sent to the predefined numbers through GSM. RF transmitter sends signal to the RF receiver through RF network. When the RF receiver receives signal from the transmitter it turns on the buzzer in the transmitter module. The architecture of the transmitter consists of ATMEGA 328P microcontroller which is the main source. It gets input signals from the comparator when the connection flow between the ends of the thread is terminated by breaking the thread. Then it gives input to the GSM and RF transmitter. The GSM module that is described in the transmitter module is SIM900A, it sends alert message and makes emergency call to the pre-set numbers. The RF transmitter sends signal to RF receiver through RF network. The receiver module architecture consists of nano ATMEGA 328P microcontroller. It gets its input signal from $\mathrm{RF}$ receiver. Once it gets the signal it turns on the buzzer.

\section{B. Methodology}

This Methodology commences with a brief statement enumerating the working of hardware and software in this proposed module. This module ensures emergency calls and alert message along with the location of the victim to the predefined numbers. When a woman feels that she is under threat she can activate the device through HC-05 Bluetooth module which is a software-controlled device. It can be controlled through mobile phone. Once the device is activated, If the woman is attacked, she can simply break the thread which is stitched along with her dress. The connectivity between either end of the thread is continuously monitored by the LM 315 comparator. When the connection between the ends of the thread is broken by the victim the comparator it sends signal to the ATMEGA328P microcontroller to which the comparator is connected. ATMEGA328P microcontroller is fussed with GSM and RF transmitter in the transmitter module. When the microcontroller receives signal from the comparator it in turn turns on the GSM module and RF transmitter. The GSM module makes emergency call and sends alert message along with the location of the victim to the numbers that is programmed in it. The RF transmitter sends signal to the RF receiver in the transmitter module through RF networking. The main advantage of using RF module is that it consumes no power when it is in off condition and its does not require any manual operation to get connected with the receiver. The RF receiver is connected with the buzzer through ATMEGA 328P microcontroller in the receiver module. When the RF receiver receives the signal from rf transmitter it in turn turns on the buzzer. The GPS module and GSM module are connected with ATMEGA 328P micro controller by GSM AT commands and GPS AT commands using ATMEGA programmer.

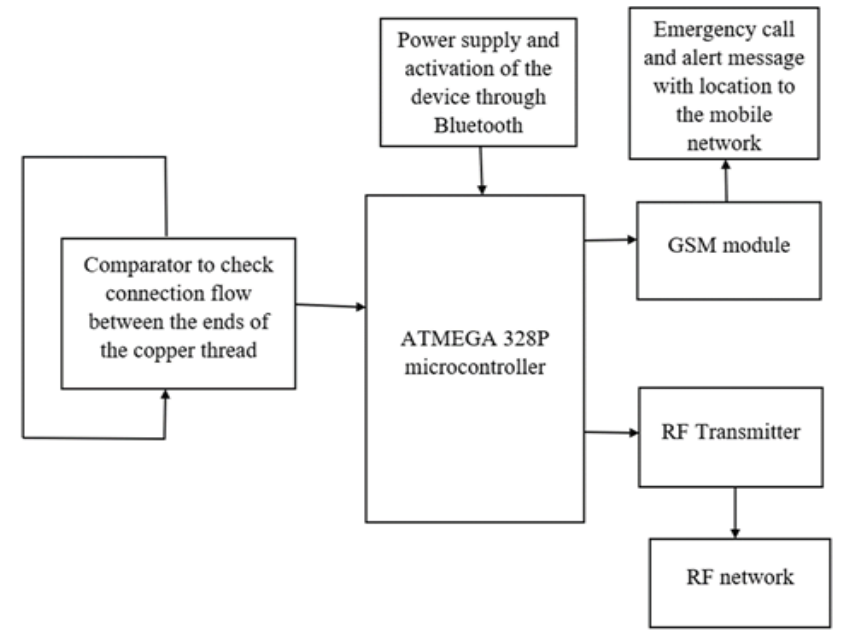

Fig. 1. Transmitter Block Diagram

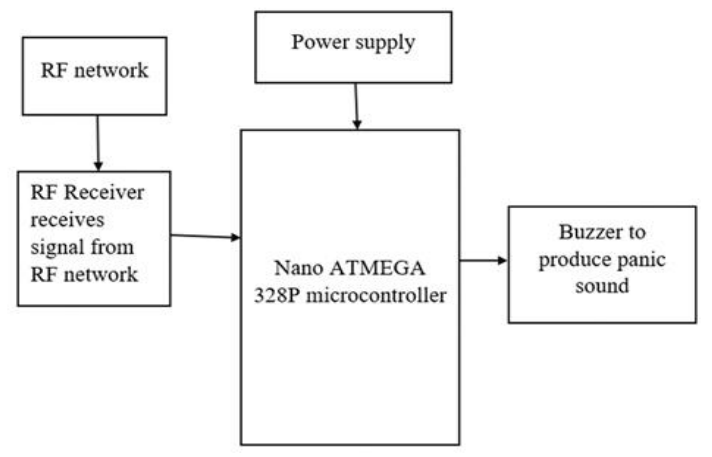

Fig. 2. Receiver Block Diagram

\section{Flow Chart}

The description of the transmitter module flow chart is given here. The device is $\mathrm{ON}$ when it gets connected to power supply. The device is activated through Bluetooth. Once the device is activated the comparator continuously checks for the connection flow between the thread. Once the connection flow is terminated. The comparator pushes the microcontroller to turn on the GSM module and RF transmitter. The GSM module makes emergency call and sends alert message to the 
predefined number. RF transmitter sends signal to the RF receiver through $\mathrm{RF}$ network.

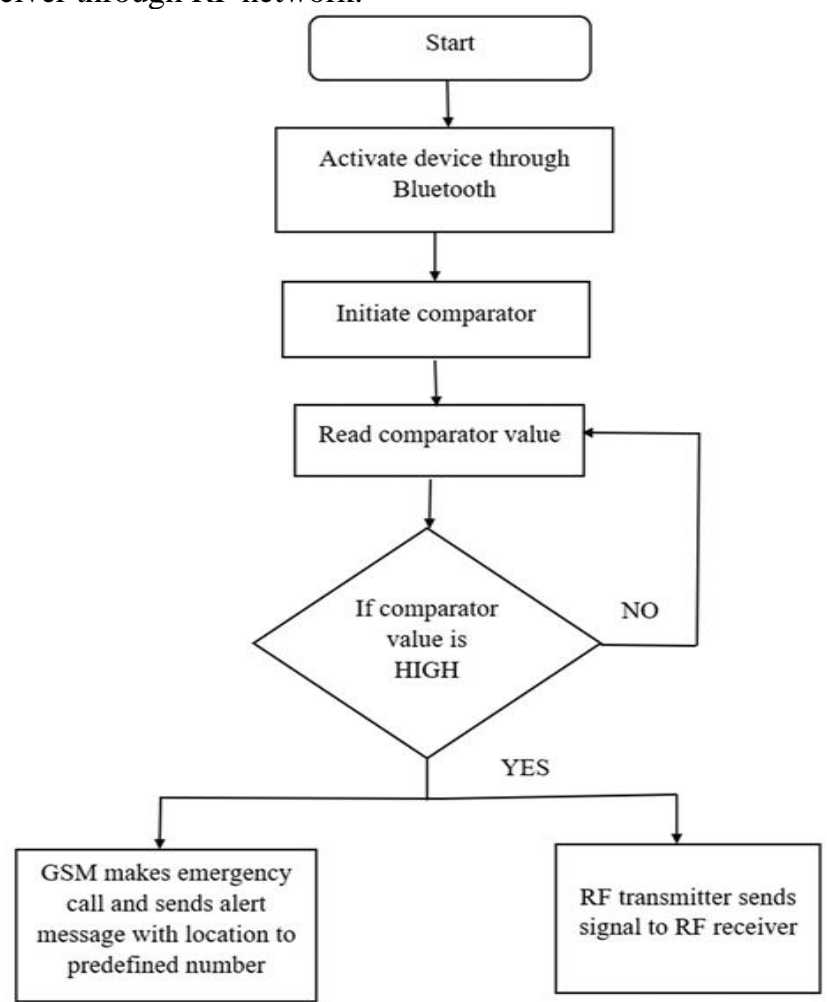

Fig. 3. Transmitter Flow Chart

The device is ON when it gets connected to power supply. $\mathrm{RF}$ receiver receives signal from the RF transmitter through RF network. It passes the signal to the microcontroller. Once the microcontroller receives the signal it immediately turns on the buzzer to produce sound.

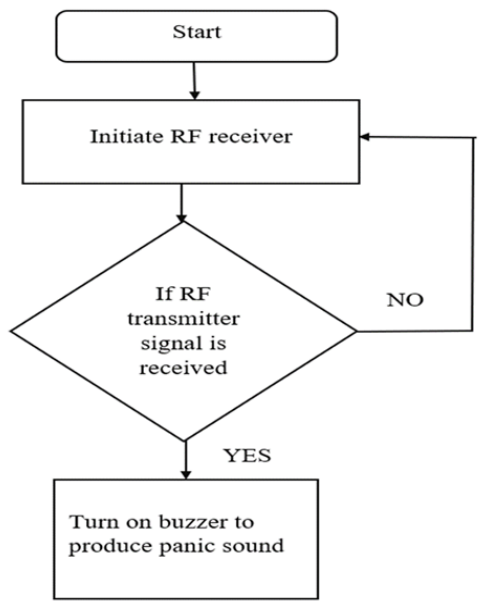

Fig. 4. Receiver Flow Chart

III. RESULT

The proposed system continuously monitors the connection flow between the ends of the thread when the device is activated through Bluetooth. When the connection is terminated by breaking the thread by the victim during distress situations or when she feels that she is under threat, the system makes emergency call and sends alert message along with the longitude and latitude position of the victim to the predefined numbers through GSM module at transmitter module. The buzzer is turned on to produce sound which acts as panic attack to the intruder at the receiver module.

When the connection between the ends of the thread is terminated, the transmitter module makes emergency call and sends alert message along with location of the victim to the predefined numbers which is shown in Fig. 5 and Fig. 6 and the receiver module blows the buzzer to produce panic sound.

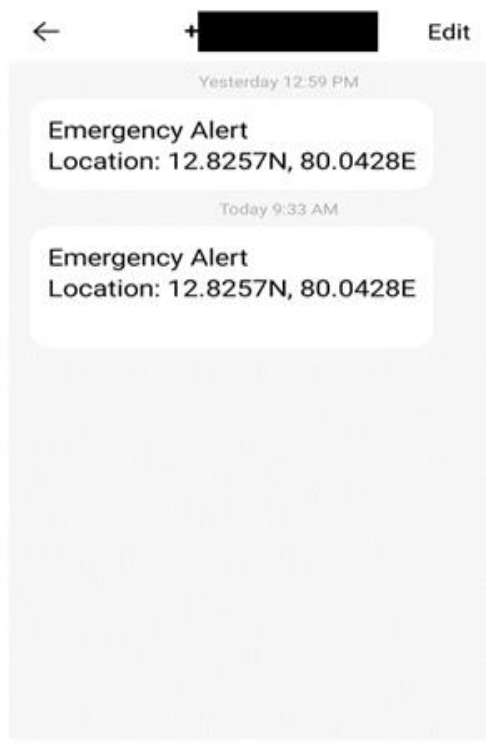

Fig. 5. Alert message along with location to predefined number

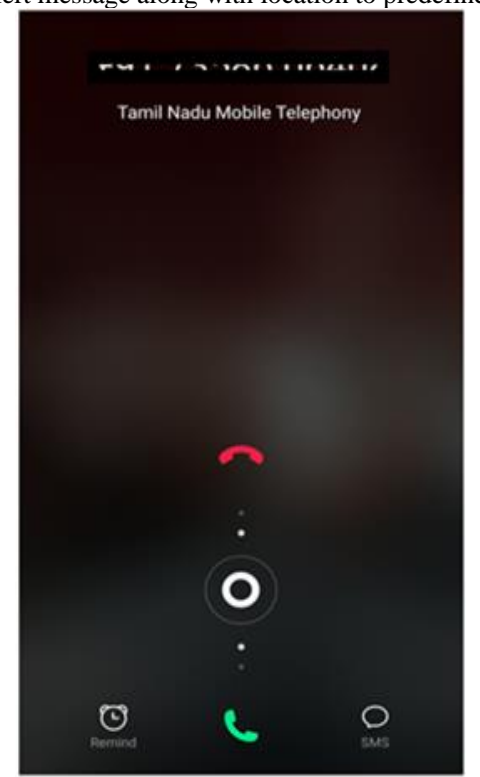

Fig. 6. Emergency call to predefined number

\section{CONCLUSION}

Appealing to the requirement of women security outside the home, many approaches of providing security to user through mobile application and safety devices have been explored but the main drawback on them are they use various sensors for monitoring their physiological conditions, at times which may lead to false alert. The proposed system consists of separate transmitter and receiver module. Copper thread along with the transmitter module is stitched along with dress. At distress situation if the thread broke by the victim the system makes emergency call and alert message to the predefined numbers 
through GSM and the buzzer is turned on. The error probability is reduced by not using any sensors. It is more accurate when compared to existing system. This device will be very useful to women.

\section{REFERENCES}

[1] M.S. Bhuvaneswari, A. Shirly Edward and S.G. VijayaKumari, "GSM Based Women's Safety Device”, International Journal of Pure and Applied Mathematics

[2] U. Jaishree Vidhya, V.K. Jothi Sree, T. Pratheeba, K. Ragapriya and B Sathyasri, "Design and implementation of Women Safety System based on IoT technology", International Journal of Recent Technology and Engineering

[3] Kalpana seelam and K. Prasanti, "A novel Approach to Provide Protection for Women by using smart security device", Proceeding of the IEEE second International Conference on Inventive Systems and Control.

[4] Mahejabeen Budebhai "IoT based child and women safety", International Journal of Computer science and Mobile Computing.

[5] D.G. Monisha, M. Monisha, G. Pavithra and R. Subhashini, "Women safety device and application - FEMME" Published an article on Indian Journal of Science and Technology on March 2016.

[6] G.C. Harikiran, Karthik Menasinkai and Suhas Shirol, "Smart Security solution for women based on IoT", International Conference on Electrical, Electronics and Optimization Techniques (ICEEOT)-2016.

[7] S. Anu Roopa Devi, M. Ganesh Kumar, R. Hari Sudhan, P. Sathiya and A. Udhaya Prakash, "Arduino ATMEGA-328 microcontroller", International Journal of Innovative Research in Electrical, Electronics, Instrumentation and Control Engineering (IJIREEICE).

[8] Nitish Aggarwal and Nishant Bhardwaj. (2014), "Design and Development of "Suraksha"-A Women Safety Device", International Journal of Information \& Computation Technology. 